UDC 591.398: 612.821.2: 616-089.811

Tsupykov O. M. ${ }^{1,2,3}$, Kyryk V. M. ${ }^{2}$, Rybachuk O. A. ${ }^{1,2,3}$, Poberezhnyi P. A. ${ }^{2}$, Mamchur A. A. ${ }^{2}$,

Butenko G. M. ${ }^{2}$, Pivneva T. A. ${ }^{1,2,3}$, Skibo G. G. ${ }^{1,2,3}$

${ }^{1}$ Bogomoletz Institute of Physiology NAS Ukraine, Kyiv, Ukraine

${ }^{2}$ State Key Laboratory, Kyiv, Ukraine

${ }^{3}$ State Institute of Genetic and Regenerative Medicine NAMS Ukraine, Kyiv, Ukraine

e-mail: oleg_tsupikov@mail.ru

\title{
EFFECT OF NEURAL STEM CELL TRANSPLANTATION ON COGNITIVE FUNCTIONS OF MICE AFTER CEREBRAL ISCHEMIA-REPERFUSION
}

\section{ABSTRACT}

This study is aimed to determine the effect of transplantation of neural progenitor cells (NPCS) isolated from fetal hippocampus on cognitive functions of experimental animals after short-term global cerebral ischemia. NPCs were isolated from hippocampus of $F V B$-Cg- $T g(G F P U) 5 N a g y / J$ mice, transgenic by the GFP. Ischemic brain injury in FVB «wild» type mice was modeled by bilateral occlusion of the common carotid arteries for 20 min. GFP-positive NPCS were stereotaxically transplanted into the hippocampus of experimental animals in 24 hours after ischemiareperfusion. Cognitive functions were evaluated using Morris water maze. Results of this study showed that global short-term cerebral ischemia resulted into cognitive impairments in mice. Stereotaxic transplantation of NPCs promoted the cognitive function recovery in experimental animals after ischemic brain injury. Thus, the data indicates that transplantation of NPCs may have a therapeutic effect in treating of ischemic stroke.

KEYWORDS: cerebral ischemia, hippocampus, cognitive functions, neural stem cells, transplantation.

Ischemic brain lesions are one of the leading causes of disability and mortality in the elderly. According to WHO, stroke is the second most frequent, after cardiovascular diseases, cause of death. Due to the increased level of morbidity from atherosclerosis, hypertension, diabetes mellitus, stroke is more common in people of working age [12]. Severe hypoxic-ischemic encephalopathy in newborns can cause the development of cerebral palsy [22]. It is known that cerebral stroke leads to a number of emotional disorders (irritability, increased excitability and anxiety, etc.) [3]. In experimental models of global cerebral ischemia has been shown that ischemic brain damage in animals causes the memory and learning ability disorders, hyperthermia, and a significant increase in locomotor activity $[1,14,23]$.

It is believed that the effects of ischemic damage on nervous tissue can be compensated by activation of their own reparative mechanisms or correction through various therapeutic methods [18]. Recently the possibility of using stem cell therapy for the treatment of ischemic and degenerative diseases of the neural system has been actively studied [28]. It is planned to use stem cells from different sources and various differentiation potential (from embryonic and fetal cells to the cells of an adult organism), as an active agent $[19,25]$. The basis of these studies is the theoretical and experimental developments that indicate the ability of stem cells to differentiate into neuronal or glial cells, compensating for lost tissue function. For example, the use of fetal neural stem cells (NSCs) suggests their differentiation into specialized cells of the central neural system $[8,13]$. During embryogenesis, NSCs are located in the ventricular zone of the neural tube, and are able to differentiate into all cell types, which are necessary for the formation of the CNS. It was thought that neurogenesis occurs only during embryonic development of an organism. Recent studies have shown that new neurons are continuously generated from the NSCs throughout life [7, 29]. This neurogenesis in adult mammals occurs in certain areas of the brain, namely subgranular zone (SGZ) of hippocampal dentate gyrus and subventricular zone (SVZ) of lateral ventricles.

Also we know that stem cells are able to recognize areas of damaged tissue, migrate toward these areas, and differentiate into cell type needed to restore lost function $[6,11,30,31]$. To investigate the possible regenerative potential of NSCS there are widely used various models of ischemic brain damage with subsequent transplantation [7, 16, 21].

However, the introduction of stem cell transplantation methods into clinical practice should be based on a profound understanding of their functioning mechanisms, and sufficient experimental data. The study of selected range of issues is possible under experimental transplantation involving adequate models of pathology in laboratory animals. Experimental ischemic brain lesions in mice allow simulating 
the effects of ischemic stroke in humans $[4,9,10]$. Transplantation of cells of different origin with various differentiations potential aims at study of their regenerative potential, and general functioning principles in restorative processes after ischemic damage.

Considering all of the above, the aim of this study was to investigate the effect of fetal neural progenitor cells transplantation on cognitive function of experimental animals after short-term global cerebral ischemia.

\section{MATERIALS AND METHODS}

All experiments were performed in accordance with international principles of the European Convention for the protection of vertebrate animals used for experimental and other scientific purposes (European convention, Strasburg, 1986), article 26 of the Law of Ukraine «On protection of animals from cruelty» (№ 3447-IV, 21.02.2006), and all norms of bioethics and biosafety.

In our study, we used FVB «wild» type and FVB-C-Tg(GFPU)5Nagy/J mice, transgenic by the green fluorescent protein gene (GFP). The mice were kindly provided by the European Molecular Biology Laboratory (Monterotondo, Italy).

Pubertal male FVB «wild» type mice (12 weeks) were randomly assigned to one of three groups: A - control $(n=5)$ - sham-operated animals without ischemia/reperfusion injury and neural progenitor cells transplantation (NPCS); B - mice $(\mathrm{n}=13)$ with NPCs transplantation after ischemia/reperfusion; and $C$ - animals ( $n=5)$, which after ischemia/ reperfusion were injected with the NPCs's cultivation medium.

\section{ISOLATION AND CULTURE OF NPCS}

The FVB-Cg-Tg(GFPU)5Nagy/J mice, transgenic by GFP gene, were used as donors of neural progenitor cells. The source of NPCS was hippocampus isolated in sterile conditions from the fetus brain on 17-18 days of embryonic development. Fetal nervous tissue was mechanically dissociated in the Neurobasal medium with Pasteur pipettes of different diameters. After the dissociation, a cell suspension was passed through nylon cell strainer (Falcon, USA) with a pore diameter of $40 \mu \mathrm{m}$. A purified fraction of NPCS was obtained by centrifugation of cell suspension in density gradient (22\% solution of Percoll). The percentage of viable cells in suspension was determined by flow cytometry, using a cell sorter FACSAria (Becton Dickinson, USA), after incubation of cell suspension with 7-amino-actinomycin $D(7-A A D)$.

To analyze the phenotype of NPCS there was used an immunocytochemical staining of cells in culture. For this purpose, freshly isolated cells were seeded on cover glasses coated with Matrigel $\left(4 \cdot 10^{5}\right.$ cells per 35-mm cultural dish). NPCs were cultured in Neurobasal medium (Invitrogen, USA) with $20 \mathrm{ng} / \mathrm{ml}$ human recombinant FGF-2 (R\&D). On the third day of cultivation, the culture medium was replaced with cold of $4 \%$ paraformaldehyde (PFA), and after fixation immunocytochemical staining of cell culture was performed.

\section{IMIMUNOCYTOCHEMICAL STAINING OF NPCS CULTURE}

After washing off the PFA, cells culture was blocked in a $0,1 \mathrm{M}$ phosphate buffer $(\mathrm{pH}=7,4)$ supplemented with $0,5 \%$ bovine serum albumin $(B S A)$ and $0,3 \%$ Triton $X-100$. Marker of neural progenitor cells nestin was used to characterize the phenotype of the cell culture. For this purpose cell culture was incubated in a solution of primary mouse anti-nestin monoclonal antibodies (Chemicon, USA) for 12 hours at $4{ }^{\circ} \mathrm{C}$. Primary antibodies were visualized by anti-mouse secondary antibodies conjugated with fluorochrome Alexa Fluor 555 (Invitrogen, USA). The nuclei of cells were contrasted with fluorescent dye Hoechst33342 (Invitrogen, USA). Dyed cell culture was covered with medium Immu-
MOUNT (Thermo Scientific, USA). Immnocytochemicaly stained culture of NPCs was analyzed using a confocal scanning microscope FV1000BX61WI (Olympus, Japan).

\section{MODELING OF HIPPOCAMPAL ISCHEMIC INJURY}

Ischemic injury of the hippocampus in FVB «wild» type mice was modeled by bilateral occlusion of the common carotid arteries for $20 \mathrm{~min}$. The animals were anesthetized with 2,2,2-tribromoethanol (125$240 \mathrm{mg} / \mathrm{kg}$, intraperitoneally). Then, under the surgery stereomicroscope, the common carotid arteries were isolated; and atraumatic elastic vascular micro-clamps were applied on them. After 20 minutes the clips were removed and the wound was sutured in layers under aseptic condition. In sham-operated animals of the control A group only artery preparation was done and they were under anesthesia for 20 minutes without application of clips.

\section{MEASUREMENT OF REGIONAL CEREBRAL BLOOD FLOW}

To assess a regional cerebral blood flow (rCBF) and confirm ischemic conditions after bilateral occlusion of the carotid arteries there was used a laser Doppler flowmeter (moor-VMS-LDF-1, Moor Instruments, UK). $r C B F$ was controlled before and during occlusion, then straight after reperfusion. The data were analyzed using the software moorLAB (Moor Instruments, UK). In next studies, we used only animals, which $r C B F$ fell below $15 \%$ of normal baseline before occlusion.

\section{TRANSPLANTATION OF HIPPOCAMPAL NPCs}

Syngeneic transplantation of 2-2,5•10 GFP-positive NPCs in volume of $2 \mu \mathrm{l}$ of medium were injected into the hippocampus of animals with ischemic model stereotaxically (coordinates from the bregma: lateral $\pm 1,5 \mathrm{~mm}$, posterior $-2,0 \mathrm{~mm}$, dorsoventrally $1,7 \mathrm{~mm}$ ) under combined 2,2,2-trybromoethanol anesthesia ( $125 \mathrm{mg} / \mathrm{kg}$, intraperitoneally). Shamoperated animals were injected with the NPC's culture medium in the same location.

\section{BEHAVIORAL TEST}

To assess cognitive functions (spatial memory) there was used the Morris water maze (MWM). The role of $M W M$ performed a black round pool (150 cm in diameter and $60 \mathrm{~cm}$ height), containing transparent plexiglass platform [24]. During the experiment the platform remained in a constant location. Water temperature was $28^{\circ} \mathrm{C}$. The walls of the pool were marked with high contrast geometric images (squares, stars, triangles). Such external visual cues contributed to the formation of the idea about spatial location of platform in animals. The trajectory and time of animal swimming were monitored with a digital video camera. Testing of animals in the to check their ability of executing the tasks related to spatial memory was performed according to the procedure adapted for mice [20]. For four consecutive days (from 12 to 15 days after ischemia) there were performed behavioral tests. During these tests the mice were trained to find the platform, oriented on the external visual cues. Each session consisted of 2 cycles ( 4 attempts) per day, during which the mice were placed into the pool, and had 60 seconds to find the platform. For each animal of all experimental groups there was determined a parameter «time of platform searching» (an average of 8 attempts at the time, in seconds), spent by the mouse to find the platform in a particular day of the study. If the mouse did not find the platform within 60 seconds, it was placed on the platform for memorizing its location. On the fifth day of testing (16 days after ischemia), transparent platform was submerged for a few millimeters from the water surface; and each mouse was placed into the pool to find a hidden platform. 


\section{STATISTICAL ANALYSIS}

Statistical data analysis was performed, using the software Statistica (version 5, StatSoft). Values are presented as mean value \pm standard error of the mean. Nonparametric Kolmogorov-Smirnov test was used to assess differences between the values.

\section{RESULTS AND DISCUSSION}

To study the phenotype of hippocampal neural progenitor cells culture, we used the immunocytochemical method. Immunocytochemical analysis showed that at the third day of cultivation NPCs were mostly rounded or had bipolar shape and thin spines. The majority of cells $(95,2 \%)$ expressed marker of immature cells - nestin (Fig. 1), which is typical for type-2 hippocampal progenitor cells [17]. These data indicate that the cells, which we used for transplantation, had properties of neural progenitor cells.

The effect of ischemic brain injury on cognitive function and possible neuroprotective effect of neural stem cells transplantation in our study were assessed, using behavioral tests - Morris water maze.

The animals from each experimental group were tested for 5 days from the $12^{\text {th }}$ day after surgery. The time spent on searching the platform during the first 4 days of testing decreased in animals of all experimental groups. Despite the reduction in time spent to search the platform in all groups, this parameter in the group of animals with ischemia was significantly greater than that in sham-operated mice (control) and in the group of animals with ischemia plus NPCs transplantation. In animals of the control group time of platform search was $35,3 \pm 3,4$ sec on the $12^{\text {th }}$ day, and then decreased to $20,3 \pm 2,1 \mathrm{sec}$ on the $15^{\text {th }}$ day (Fig. 2). Compared with the control, ischemic animals had higher scores, indicating a lack of spatial memory and learning ability. The time of platform search in this animal group also decreased from $12^{\text {th }}$ day to $15^{\text {th }}$ day and reached $48 \pm$ 2,7 sec and $32 \pm 2,3 \mathrm{sec}$, respectively (Fig. 2).

In the group of animals with NPCs transplantation after ischemic brain damage more rapid regression of cognitive impairment was observed, including reduce of the deficit of spatial memory and learning ability. Search time in this group decreased from $12^{\text {th }}$ day to $15^{\text {th }}$ day and was 42 $\pm 1,5 \mathrm{sec}$ and $22 \pm 1,0 \mathrm{sec}$, respectively (Fig. 2).

On the $16^{\text {th }}$ day, when the platform was submerged under the water, the time of platform search increased in all groups of animals, and amounted $24,3 \pm 2,7,36 \pm 2,3$ and $28,8 \pm 1,4 \mathrm{sec}$ in control, ischemic and the group with ischemia plus NPCs transplantation, respectively. There were no statistically significant differences between control animals and a group of «transplant ischemia plus NPCS» at all time intervals.

To assess cognitive function of experimental animals there have been developed many behavioral tests, but the water maze, designed by Richard Morris, is one of the most widely used behavioral tests for studying spatial learning and memory [24, 27]. Successful execution of the MWM test by animals is associated with long-term potentiation (LTP) and NMDA-receptor function, making this test the basic approach in the study of hippocampal neural networks $[2,15]$. Our results indicate that short-term global cerebral ischemia resulted in a disorder of spatial learning in experimental animals. We observed recovery of cognitive functions in two weeks after NPCs transplantation. Even if two weeks is sufficient for differentiation of transplanted progenitor cells into neurons and glial cells this term is not sufficient for donor cells integration into existing neural hippocampal networks of the recipient [26]. We consider that recovery of spatial learning and memory in experimental animals may be due to the secretion of trophic and mitogenic factors of the transplanted cells, which contributed to the survival of damaged cells and restoration of their lost functions. Several studies have shown that restoration of ischemic neurological status of animals in the early period after stem cell transplantation is likely not due to the replacement of damaged cells with new transplanted ones but to donor cell secretion of various growth factors [5, 32].

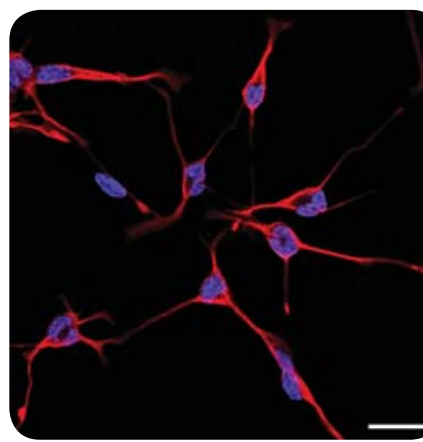

4

Fig. 1. Immunocytochemica staining of neural stem cells culture for nestin. Most cells were positive for nestin (red). Nuclei were contrasted by Hoechst 33342 (blue). Scale $=20 \mu \mathrm{m}$.

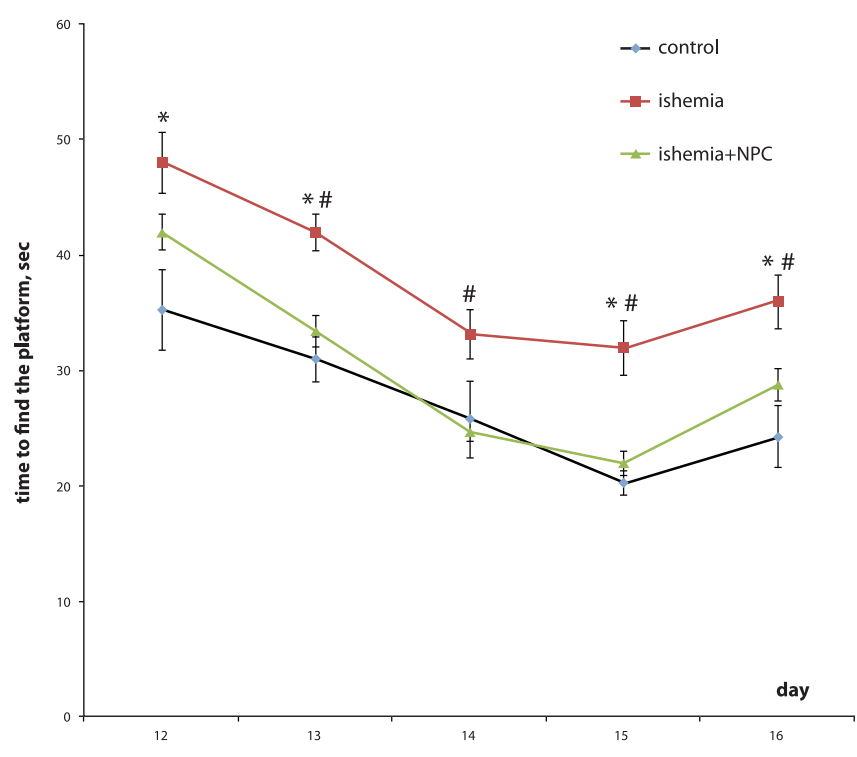

Fig. 2. The time of platform search in the Morris water maze. The chart shows the average time of platform search by sham-operated animals (control), ischemic mice (ischemia), and group of ischemic animals with transplanted NPCs (). * $-p<0.05$ compared with control group, \# $-p<0,05$ compared with the group of animals with ischemia plus NPCs transplantation.

\section{CONCLUSIONS}

THUS, STEREOTAXIC TRANSPLANTATION OF NEURAL PROGENITOR CELLS CONTRIBUTES TO THE RESTORATION OF COGNITIVE FUNCTION (SPATIAL MEMORY) IN ANIMALS AFTER ISCHEMIC BRAIN INJURY, MODELED BY BILATERAL OCCLUSION OF THE COMMON CAROTID ARTERY. 


\section{REFERENCES}

1. Babcock A. M., Baker D. A., Lovec R. Locomotor activity in the ischemic gerbils // Brain Res. - 1993. - 625. - P. $351-354$.

2. Bannerman D. M., Good M. A., Butcher S. P. et al. Distinct components of spatial learning revealed by prior training and NMDA receptor blockade // Nature. - 1995. 378. - P. 182-186.

3. Caeiro L., Ferro M.J., Albuquerque R. et al. Delirium in the first days of acute strokeJ // Neurol. - 2004. - 251. - P. 171-178.

4. Carmichael S.T. Rodent models of focal stroke: size, mechanism, and purpose // NeuroRx. - 2005. - 2, № 3. - P. 396-409.

5. Chen J., Zhang Z. G. Li Y. et al. Intravenous administration of human bone marrow stromal cells induces angiogenesis in the ischemic boundary zone after stroke in rats // Circ. Res. - 2003. - 4. - P. 692-699.

6. Darsalia V., Kallur T., Kokaia Z. Survival, migration and neuronal differentiation of human fetal striatal and cortical neural stem cells grafted in stroke-damaged rat striatum // Eur. J. Neurosci. - 2007. - 26. - P. 605-614.

7. Gage F. H. Transplantation in the future // Prog. Brain Res. - 2012. - 201. - P. 7-13.

8. Gelati M.,Profico D., Projetti-Pensi M. et al. Culturing and expansion of «clinical grade» precursors cells from the fetal human central nervous system // Methods Mol. Biol. - 2013. - 1059. - P. 65-77.

9. Ginsberg M.D., Busto R. Rodent models of cerebral ischemia // Stroke. - 1989. - 20, № 12. - P. 1627-1642.

10. Graham S. M, McCullough L. D, Murphy S. J. Animal Models of Ischemic Stroke: Balancing Experimental Aims and Animal Care // Comp. Med. - 2004. - 54, № 5. - P. 486 - 496.

11. Guzman R., Bliss T., De Los Angeles A. et al. Neural progenitor cells transplanted into the uninjured brain undergo targeted migration after stroke onset // J. Neurosci. Res. - 2008. - 86. - P. $873-82$.

12. Hadjiev D. I., Mineva P. P, Vukov M. I. Multiple modifiable risk factors for first ischemic stroke: a population-based epidemiological study // Eur. J. Neurol. - 2003. 10, № 5. - P. 577 - 582.

13. Hsu Y. C., Lee D. C., Chiu I. M. Neural stem cells, neural progenitors, and neurotrophic factors // Cell Transplant. - 2007. - 16, № 2. - P.133-150.

14. Ide T., Morikawa E., Kirino T. An immunosuppressant, FK506, protects hippocampal neurons from forebrain ischemia in the Mongolian gerbil // Neurosci. Lett. - 1996. 204. - P.157-160.

15. Jeffery K. J.,Morris R. G. Cumulative long-term potentiation in the rat dentate gyrus correlates with, but does not modify, performance in the water maze // Hippocampus. 1993. - 3. - P. 133-140.

16. Jenny B., Kanemitsu M., Tsupykov O. et al. Fibroblast growth factor-2 overexpression in transplanted neural progenitors promotes perivascular cluster formation with a neurogenic potential // Stem Cells. - 2009. - 27, № 6. - P. 1309-1317.

17. Kempermann G., Jessberger S., Steiner B., Kronenberg, G. Milestones of neuronal development in the adult hippocampus // Trends Neurosci. - 2004. - 27. - P. 447-452.

18. Kollmar R., Schwab S. Ischaemic stroke: acute management, intensive care, and future perspectives // Br. J. Anaesth. - 2007. - 99, № 1. - P. 95-101.

19. Kondziolka D., Wechsler L., Goldstein S. et al. Transplantation of cultured human neuronal cells for patients with stroke // Neurology. - 2000. - 55, № 4. - P. 565-569.

20. Lelong V., Dauphin F., Boulouard M. RS 67333 and D-cycloserine accelerate learning acquisition in the rat // Neuropharmacology. - 2001. - 41. - P. 517-522.

21. Lindvall O., Kokaia Z. Stem cells for the treatment of neurological disorders // Nature. - 2006. - 441, № 7097. - P. $1094-1096$.

22. Marret S., Vanhulle C., Laquerriere A. Pathophysiology of cerebral palsy // Handb. Clin. Neurol. - 2013. - 111. - P.169-176.

23. Matsuda S., Wen T.-C., Morita F. et al. Interleukin-6 prevents ischemia-induced learning disability and neuronal and synaptic loss in gerbils // Neurosci. Lett. - 1996. 204. - P. 109-112

24. Morris $R$. Developments of a water-maze procedure for studying spatial learning in the rat // J Neurosci Methods. - 1984. - 11. - P. 47-60.

25. Savitz S. I., Dinsmore J. H., Wechsler L. R. et al. Cell therapy for stroke // NeuroRx. - 2004. - 1, № 4. - P. 406-414.

26. Tsupykov 0. M., Pivneva T. A., Poddubna A. 0. et al. Migration and differentiation of transplanted fetal neurogenic cells in animals with brain ischemia // Fiziol. Zh. 2009. - 55, № 4. - P. 41-49.

27. Vorhees C. V., Williams M. T. Morris water maze: procedures for assessing spatial and related forms of learning and memory // Nat. Protoc. - 2006. - 1, № 2. P. 848-58.

28. Williams B. A., Keating A. Cell therapy for age-related disorders: myocardial infarction and stroke - a mini-review // Gerontology. - 2008. - 54, № 5. - P. 300-311.

29. Wong A. M., Hodges H., Horsburgh K. Neural stem cell grafts reduce the extent of neuronal damage in a mouse model of global ischaemia // Brain Res. -2005 . - 1063, № 2. - P. 140-50.

30. Yin W., Ma L., Zhang J. et al. The migration of neural progenitor cell mediated by SDF-1 is NF-KB/HIF-1 a dependent upon hypoxia // CNS Neurosci. Ther. - 2013. - 19, № 3. - P. 145-53.

31. Yuan T., Liao W., Feng N. H. et al. Human induced pluripotent stem cell-derived neural stem cells survive, migrate, differentiate, and improve neurological function in a rat model of middle cerebral artery occlusion // Stem Cell Res. Ther. - 2013. - 4, № 3. - P. 73-83.

32. Zhao L. X., Zhang J., Cao F. et al. Modification of the brain-derived neurotrophic factor gene: a portal to transform mesenchymal stem cells into advantageous engineering cells for neuroregeneration and neuroprotection // Exp. Neurol. - 2004. - 4. - P. 396-406. 